\title{
Sports Equipment Rental System of School Based on B/S Structure Design and Implementation
}

\author{
Yanxiu Wang ${ }^{1,}$, Yipai Jiang ${ }^{2}$ \\ ${ }^{1}$ School of Humanities And Social Sciences, Jingdezhen Ceramic Institute, Jingdezhen, Jiangxi, \\ China
}

ajyp333000@126.com

Keywords: Software Engineering, B/S Architecture, Equipment Rental, Equipment Management

\begin{abstract}
The school's sports equipment management center has run the sports equipment management in long-term. It was on manual paper records to record sports equipment storage, do statistics, lend, and get back. It cannot get the sports equipment timely statistical information, and cannot record the information. So a simple and easy solution effective was needed. The main subject of the research significance: We plan to build a suitable school sports equipment hiring management systems. It has analyzed the system architecture and design patterns, and analyzed the system using J2EE development platform and B / S architecture. The equipment hiring system should include the data entry module, the equipment online query module, online reservation module and statistics module. It learns the functional requirements of the system, the database structures, and the system features modular and architecture design. We used the open resource coding platform.
\end{abstract}

\section{Introduction}

The school sports venues to shoulder the obligation that rent sports equipment. Currently we are artificial lease registration way, the specific return equipment etc. is a mess. And this kind of confusion is the result of the equipment, the use is less, can be leased equipment soon up and have to apply for funding. The technical implementation plan in the software industry, it is concluded that $\mathrm{B} / \mathrm{S}$ architecture is a software industry promoting the direction of future development, also can make our sports equipment leasing system is convenient, make more effective management process [1].

The research and application of $\mathrm{B} / \mathrm{S}$ architecture will make it popular B/S architecture is more common. B/S in the school sports in the implementation and the widespread application, will make the B/S framework related technology more perfect, from engineering strategy optimization [2].B/S structure is the base of the cloud computer, is the direction of the future software development/S architecture, will be the technical innovation and application advantages of the main researching contents of this subject [3].

$\mathrm{B} / \mathrm{S}$ structure is the trend of The Times, the Internet at home and abroad, IT light pouring into the client (browsing) camps in an attempt to bring convenient to the masses of users of all kinds of services at the same time, in the corresponding areas under siege from the pool of a piece of his own. And we borrow sports equipment lease practical problems, to confirm the B/S mode advanced at the same time, also hope to be able to thoroughly research on B/S model of all kinds of theory, proved its value, and expand its application in the field of practical [4].This topic based on B/S and related technology research, develop a based on B/S server, the browser universal application of B/S architecture, and sports equipment lease in real system to test its effectiveness in [5].The architecture and the similar structure of the difference is: as long as the communication protocol is consistent, it will ignore the client-side and server-side development language. So the client data communication protocol between the server problems is one of the difficulties of the subject $[6,7]$.

This topic of technical route is mainly based on the system requirements set project construction goal, in this project USES the Java development language, MySQL database development, Tomcat as a support platform, the system adopts B/S (browser/server) and the three layer structure of the database model. 


\section{B/S structure technology}

$\mathrm{B} / \mathrm{S}$ architecture is a kind of architecture that can receive the request passed from the Internet, and the server processes the request to respond a communication mode. This architecture allows the Internet browser users able to installation and gets the service that the application system provides .With the development of technology, a B/S structure can contain additional specified function and collaborative communication can achieve in more than one B2B applications.

$\mathrm{B} / \mathrm{S}$ can understand the relationship between the request contexts, and can produce dynamic results in every particular case .These services will base user's identity, location, and the reasons for the request to change the different handling, to create a unique, customized solution. This collaboration mechanism for those who are only interested in the final result of users, are completely transparent. B/S architecture is an interface that describes a set of operations; the operations can be visited by standard XML messages on the Internet. B/S structure can be regarded as some modular applications, these applications can be described, publish, locate and invoke on the Web.

\section{The advantages and disadvantages of $B / S$ structure technology}

Advantages: the advantages of $\mathrm{B} / \mathrm{S}$ architecture is no longer listed here refer to the above comparison of $\mathrm{B} / \mathrm{S}$ and $\mathrm{C} / \mathrm{S}$ architecture can clearly reflect the strong advantages of $\mathrm{B} / \mathrm{S}$ architecture, a development anywhere using $\mathrm{B} / \mathrm{S}$ architecture for the developers and users' biggest commitment and action.

Disadvantages: we have above the concept and advantages of B/S architecture compliment to the vertices, but "no pure gold ". B/S architecture put any business logic on the server side, the client only need a result show, this will all heavy computing power delegated to the server, bringing computing ability of the client a waste, and bringing the limited computing power server pressure. So only the $\mathrm{B} / \mathrm{S}$ architecture has strong hardware support can the server bear high users' access.

\section{The analysis of system function requirement}

System user management: the system administrator has permission to add users, delete users of the system. User permissions settings: system administrator privileges to manage user permissions. User password change: ordinary users can be done in the module code modification operations

\section{Equipment information maintenance module}

The main function of the module is the basic attribute of sports equipment leasing management system set; only Material administrator role has access to the operation.

Initialization information: system will initialize the school sports equipment to the system, the precious information is mostly excel documents and paper records, system will supply excel to upload initialization and manual entry mode to initialize existing equipment initialization information into the system. Equipment procurement: equipment procurement progress including three parts as the equipment purchasing plan, purchasing activities and equipment into the treasury. Develop a plan in the system of procurement and procurement warehousing management. Equipment of loss: to report the loss and compensation for the lost equipment situation, after the student carrying on the compensation, the loss report of the equipment management should be completed.

\section{Equipment leasing module}

The module's main function is to supervise and control the equipment leasing situation, only equipment manager roles have the authority to operate.

Equipment rent check: equipment administrators can view the rent status of all kinds of sports equipment in the system, such as basketball total 100, rent out 50, 50 remaining available. Equipment available: at the time of the teachers and students renting equipment, equipment manager need to rent out every equipment register, including what kind of equipment, who is on loan, loan is expected to 
return time, etc. Return equipment: when teachers and students return the loan of sports equipment, equipment manager will need to return the registration, ensure inventory data and system.

\section{Equipment statistical check module}

The module's main function is to sports equipment leasing management system related to statistics and view, the module is mainly directed against school leader at any time for future reference, equipment manager also have authority to operate.

Inventory check: sports equipment as the inherent assets of the school, school leaders has the right to view the known, and it is also a data support for equipment procurement. Equipment rental statistics: equipment rent situation reflects the enthusiasm of teachers and students in sports activities also facilitate more welcome to see what kind of sports equipment, need to strengthen the investment in the equipment and facilities. Equipment loss statistics: the equipment is missing reflects the status of the love when teachers and students for renting equipment, this is in order from the administrative decision-making management to support sports equipment management work. Equipment procurement statistics: the depreciation and damage of sports equipment are serious, according to the inventory situation and rent situation, have equipment without day purchasing on a regular basis. Purchase quantity and purchase of the species are recorded in the system, in favor to the transfer the cost of purchasing equipment.

\section{Three layer system architecture design}

Three layer system architecture design. In the project development, previous knowledge and combining the actual situation of the project, system is divided into three basic levels of architecture: the presentation layer, function layer and data layer.

First, here introduces the three levels, and in the presentation layer of the interaction between the user and the system. However, the current customers are mainly based on HTML browser interface. Our goal is the company's information browser unified using Internet explorer browser series of Windows system integration, without having support from install additional software application, greatly reduces the deploy of system before the deployment and maintenance workload. This test is also applicable to other browsers, such as fire fox browser. Main function layer composed of database access subroutines and domain logic. Domain logic of the application must complete all related work areas: including calculate according to the existing data input data or from the presentation layer of input data validation and performance levels are acceptable command, to determine what data should be scheduling logic. Data storage layer is mainly concerned about the durability of the data, and interact with other systems. Considering on easy extending and cost, choose open source MySQL database as the database server.

$\mathrm{B} / \mathrm{S}$ architecture server put forward the requests to $\mathrm{B} / \mathrm{S}$ structure the request processor. $\mathrm{B} / \mathrm{S}$ architecture request processor can be handled by logic written in JAVA code, of course, if in the JAVA language, has to use a JAVA language and MYSQL database connection is established the database driven class libraries, help the process to write the business data into the database and the business data from the database query need. B/S architecture the server after the reply of the request processor, again through the HTTP response to send it back to the client.

System data design .Sports equipment management system for system operation and maintenance of data management, special user account login authentication, the authentication and give corresponding operation permissions to complete the process. So the account management module as necessary and the account system with equipment information and equipment rental information is very different. System management functions including user, role, the corresponding relationship between permissions management. User management is refers to the management of user information, including the CRUD functions, and the corresponding relationship between user and role management. 


\section{System implementation and testing}

User management interface: The system administrator can manage maintenance and management for all users of the system. User management is mainly for the user to add, modify, and delete. In addition the system administrator can also manage the user role authorization, would be the operating system permissions according to different user groups.

Engineering structure system code: During system coding implementation processing, the standard directory structure should be strict observed as follows which is shown in figure 1 .

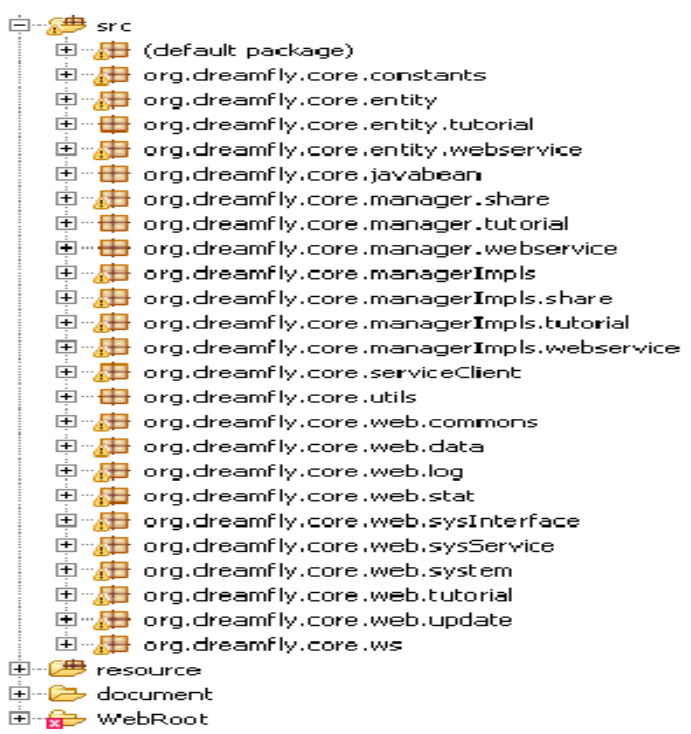

Fig.1 Source catalogue of system construction project

\section{Conclusion}

B/S structure of sports equipment rental system, the information of sports equipment and rent for unified management, and through the campus id real-name renting equipment, provide for student status management system to provide the query and the situation of students' sports equipment rental return set up a blacklist system ensure that students are more consciously in accordance with the "measures for the administration of school sports equipment spare sports equipment, timely return after using it. B/S structure of the successful implementation of sports equipment leasing system, and successfully to the function, performance evaluation, to be able to meet the pressing needs of the school sports equipment management center, can fully prove that B/S structure is excellent in the construction of digital campus and the information solutions and mature concept.

\section{References}

[1] LuoYaoZu. Network system integration and management [M]. Beijing: people's posts and telecommunications publishing house, 2005128-132

[2] Yang Yuqi. How to create a friendly user interface [J]. Microcomputer world, 1999, 2 (1) : 23-25

[3] Eric Freeman \& Elisabeth Freeman with KathySierra. Design Patterns [M]. American: O 'REILLY, 2005500-561

[4] lance Armstrong (Armstrong, e.). The Java B/S architecture Tutorial [M]. Beijing: higher education press, 2003,67-67.

[5] theo, brayton Bach. Spring in Action [M]. Beijing: people's posts and telecommunications publishing house, 2008213-275Richardson.

[6] RESTful B/S Framework [M]. Beijing: electronic industry press, 2008,78-125

[7] and rhett. The elements of user experience: Web of user-centered design [M]. Beijing: mechanical industry publishing house, 2008.113-113. 